\title{
Information Management Supporting Deployment of a Light Fieldable Laboratory: A Case for Ebola Crisis
}

\author{
Olga Vybornova*, Nicolas Dubois, Roland Gueubel, Jean-Luc Gala \\ Center for Applied Molecular Technologies, Institut de Recherche Expérimentale et Clinique, Université Catholique de Louvain, Belgium
}

Copyright $(2015$ by authors, all rights reserved. Authors agree that this article remains permanently open access under the terms of the Creative Commons Attribution License 4.0 International License

\begin{abstract}
Improved information collection, interpretation and processing are vitally important for optimized crisis response. A comprehensive information management (IM) system using ontology-based approach is aimed to handle heterogeneous information and to model the operational domain knowledge associated with the deployment of a light fieldable laboratory (LFL) during the recent Ebola crisis in Guinea. This LFL mission was part of the B-LiFE (Biological Light Fieldable Laboratory for Emergencies) project which integrates analytical and space-based technologies. LFL can be considered as a toolbox where all operational functions and related tools are incorporated into a single information space. An ontology can therefore be applied to facilitate the preparation and management of next LFL missions. The aim is to provide technical compatibility of information shared between tools and to align terminology and definitions while complying with guidelines, best practices and procedures. Accordingly, the LFL domain becomes a formalized and structured modeling of LFL concepts, procedures and functions which, in turn, helps distinguishing functions which are necessary for the mission from those which are incompatible according to a given mission or scenario. Such consistent logical modelling allows then to efficiently plan and configure any LFL mission by selecting and activating in due time, among all available functions and tools, those which are strictly mandatory.
\end{abstract}

Keywords Crisis Response, Ontology, Information Management, Knowledge Representation, Molecular Microbiology, Infectious Disease, Field Laboratory, Outbreak Response, CBRN, Field Diagnostics

\section{Introduction}

The term information management (IM) covers various stages of information processing which comprise production, storage, retrieval and dissemination of information with the aim to improve the working process [1]. Information which is key to optimized crisis response is gathered from internal and external sources, irrespective of the format [2]. In this regard, the quantity and quality of information are crucial parameters to maximize the beneficial impact of the decision-making process. Yet, as crises and related information evolve ever more dynamically, there is a need to increase the flexibility and speed of information collection, processing and storage. This includes a better accessibility and a rapid sharing of protocols, procedures and guidelines, a fast and accurate update of situational awareness, and the improvement of decision-making by all key actors. Whereas information systems need to be as comprehensive as possible, effective, simple and easy to use [3], new technology and information systems have the potential to contribute to improving response and management of disasters. However, current technology and managerial approaches dedicated to crisis response often remain ineffective due to lack of communication, interoperability and coordination. This justifies the need to develop technological approaches which take into account existing constraints while being easily usable by specific actors, and addressing the needs at the level of both headquarters and field-based operators. New approaches should enable all actors, including decision-makers, to better cope with the complexity, uncertainty and rapid changes of the crisis environment, hence to better serve the target population.

The Semantic Web describes a stack of methods and technologies which can be used to make the meaning of data more explicit. Various knowledge representation languages, such as the Web Ontology Language (OWL) or the Resource Description Framework (RDF), are used to make data available in a machine-readable and understandable way, as well as to specify ontologies that restrict the meaning of terms towards their intended representations. Taking a closer look at disaster management, it is clear that we are still far from automated interoperability; we have not yet been able to capture the tacit knowledge of the responders and translate their operational procedures or their specific domain data into a model [4].

There are various types of data generated on-site or collected from external sources, including taxonomies, 
databases, procedures, best practices and guidelines and they all consist of an extremely diverse range of subjects and formats. These data are interpreted, processed, organized, structured, and filtered to become useful information for the direct beneficiaries. Only well formatted and correctly understood information can be digested to bridge the gap between various disciplines and organizations and can be transformed into specific actions. The proper collection, processing and representation essentially decrease the risk of missing or distorting information necessary for decision-making to the benefit of affected communities.

The IM for mobile laboratories is quite a new area and it is only recently that significant efforts have been made for structuring the data, detailing all the components, specifying the information needs and gaps of users, and defining the information sharing processes between stakeholders as well as data analysis and processing in a systematic way. Among other aspects, information content, terminology and approach to data organization and presentation were recently thoroughly reviewed by mobile laboratory operators from Belgium, Germany, the Netherlands and Norway with the aim to harmonize as much as possible the concept of mobile laboratory structure [5].

The present work focuses on IM required for planning, coordination and decision-making in the specific context of rapid on-site response to a biological crisis and takes as an example the Biological Light Fieldable Laboratory (B-LiFE) project [ESA IAP-ARTES 20 project, 2012-2017]. This B-LiFE project merges the Light Fieldable Laboratory (LFL) analytical capacity with an autonomous IM infrastructure and space assets such as satellite telecommunications, satellite imagery and navigation for detailed mapping, site monitoring and geolocation.

This work presents an IM system using ontology-based approach that supports the whole cycle of operations required for B-LiFE with the aim to use it for future LFL missions [5]. Integration of these resources provides a new capacity for emergency response. It also illustrates the benefit of the ontology to plan, prepare and manage an LFL mission by enabling fusion of a wide spectrum of extremely diverse data irrespective of the type of crisis.

\section{Method}

\subsection{B-LiFE System Operational Requirements}

The role of on-site analytical laboratory stations in public health crisis is steadily increasing. The original concept of mobile laboratory was "heavy lab-on-wheels" in the context of on-site detection and identification of chemical, biological, radiological and nuclear (CBRN) threats during military operations. There is now an increasing need for rapid epidemiological survey of life-threatening infections and quick confirmatory test as illustrated in 2009 by the first deployment of a tent laboratory by a team from the Center for Applied Molecular Technologies (CTMA) in Occidental Kasai, Republic Democratic of Congo [6]. This deployment enabled to provide locally a rapid and reliable identification of monkeypox virus in patients with skin rash illness. Addressing these problems prompted to extend the LFL concept according to mission specificity and biosafety constraints, and to further develop the LFL concept as a lightweight, transportable, robust and autonomous laboratory, rapidly deployable analytical capacity close to the sampling site in case of health crisis caused by a biological agent (B-agent). Currently, LFL is designed to be operated by a rapidly deployable staff as a complementary solution to the existing network of biological fixed-site and reference laboratories. The time needed for transporting and deploying the LFL is short since the different components can be easily packaged and moved together with a limited staff of trained experts. The main advantage of deploying the LFL in close geographical proximity to the patients is its ability to provide quick results where evidence-based medical decisions need to be implemented. This was also illustrated during the last Ebola outbreak where the B-LiFE team deployed a LFL from December 2014 to March 2015 to support the medical staff of the Ebola Treatment Unit of N'Zerekore in the forest area of Guinea. As was also the case with other LFL deployments in West Africa during the same outbreak, the Belgian LFL mission demonstrated that a successful management of patients in remote areas relies on the ability to perform rapid on-site identification of Ebola viral disease (EVD) cases.

Figures 1 and 2 below provide the external view of the B-LiFE system deployed in Guinea, and give an idea of the internal view of LFL. 


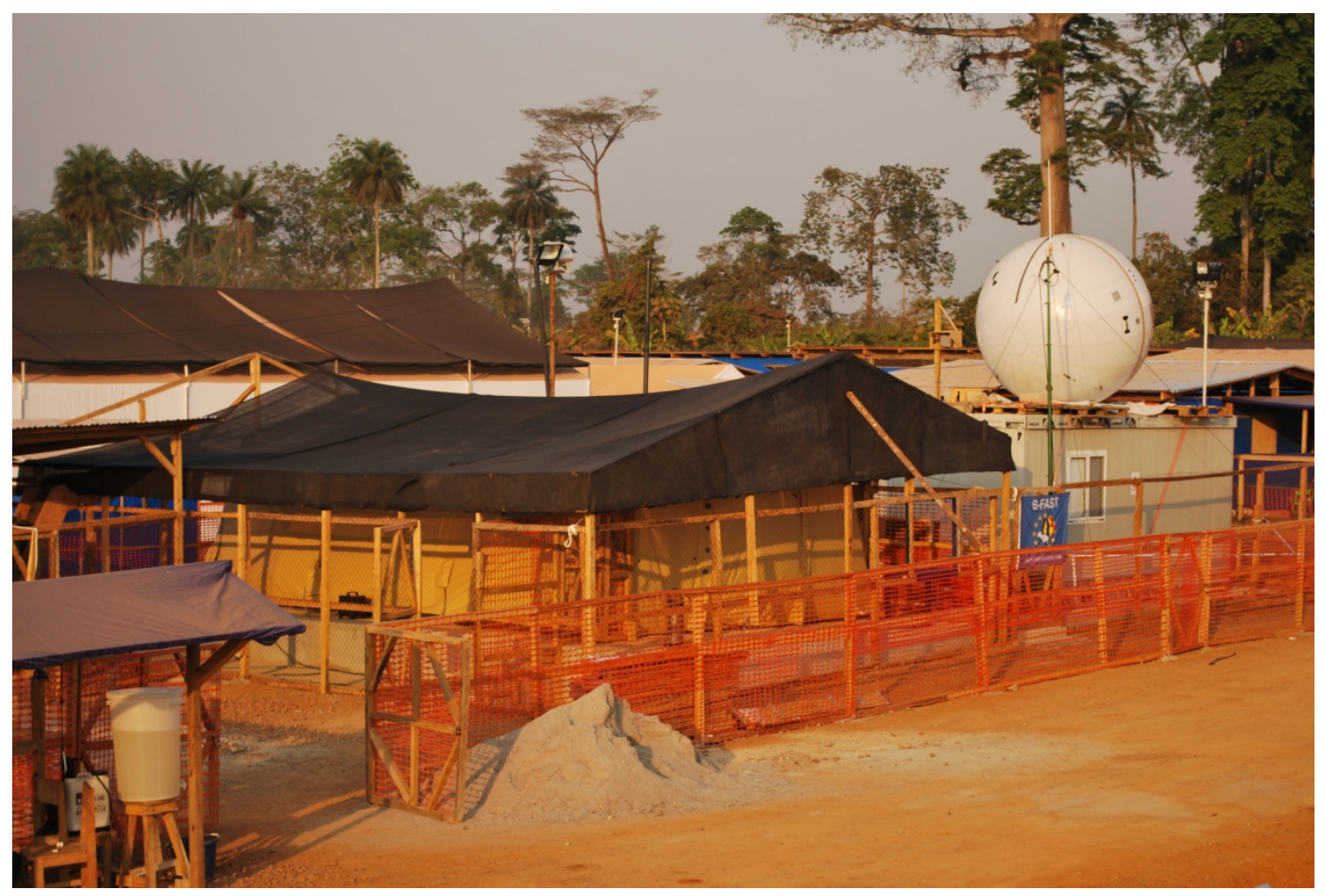

Figure 1. B-LiFE deployable analytical capacity and inflatable antenna, Ebola mission, Guinea, December 2014 - March 2015

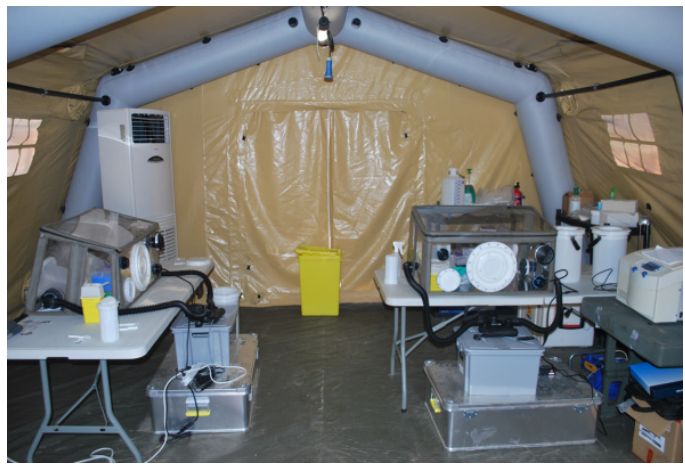

a)

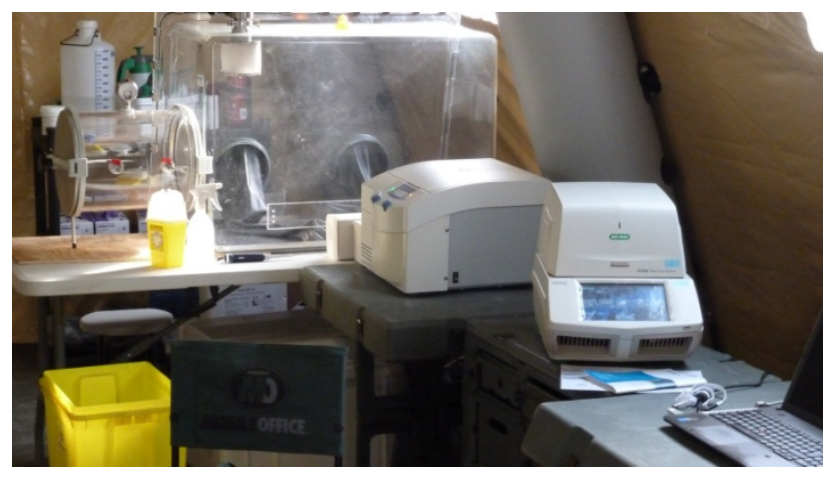

b)

Figure 2. Inside the LFL tent: a) general view, b) glovebox and analytical tools

Based on these recent missions in Africa, the many management challenges following a rapid LFL deployment have been deciphered and framed within the following major operational requirements:

- Biosafety requirements: Strict biosafety measures are taken in order to protect the laboratory staff and the neighboring population from biological threats according to the type of B-agent;

- Quality assurance: The laboratory results should be accurate, reliable and provided results rapidly to the main beneficiaries in charge of the crisis management;

- Emergency: A rapid and reliable identification of the threat is provided to first responders in the outbreak region. The faster this can be achieved, the easier the outbreak can be contained through specific countermeasures. Crisis response in the situation of emergency presumes full preparedness in advance and keeping the capacity in standby mode;

- Specificity of each mission: The type of analytical methods and biosafety measures depends on the nature of the biological threat (i.e., the type of B-agent). Parameters such the type of mission, the local context, legal dispositions, legacy and cultural specificities, and stakeholders (i.e., governmental or non-governmental organizations, international, national, and regional authorities) impact on the response configuration and planning;

- Remoteness of the bio threat location (i.e., lack of existing local facilities and poor road infrastructure) implies full autonomy of the deployed capacity, adaptability to the local conditions and mission specificities and requires an appropriate supply chain. 
In addition, remote areas are usually affected by lack of telecommunication coverage.

The LFL consists of various interlinked heterogeneous multidisciplinary components among which are shelter and analytical equipment, materials and reagents, command and control station with satellite and ICT, LFL expert and support staff, standard operational procedures (SOPs), best practices and guidelines. All these technologies and processes are tools used in specific laboratory tasks, and constitute altogether the LFL toolbox [7]. The main aim of the LFL toolbox is to detect and identify B-threats and to provide evidence-based results to beneficiaries through specific communication channels. Drawing together the integrated technology solutions into a toolbox enables users to strengthen their operational capabilities in crisis situations. Some of the best expected benefits are improved working processes, timely exchange of critical information through the whole chain of beneficiaries, increased interoperability with all stakeholders' heterogeneous multidisciplinary tools and better situational awareness. This results in better informed decision-making at each level of the crisis management process. Considering that it is impossible to foresee all potential crisis situations, the LFL toolbox is designed as generic, scalable and adaptable to different scenarios, making it more flexible and adaptable to unstable and dynamically changing environment.

The general scheme of LFL categories of tools is shown in Figure 3.

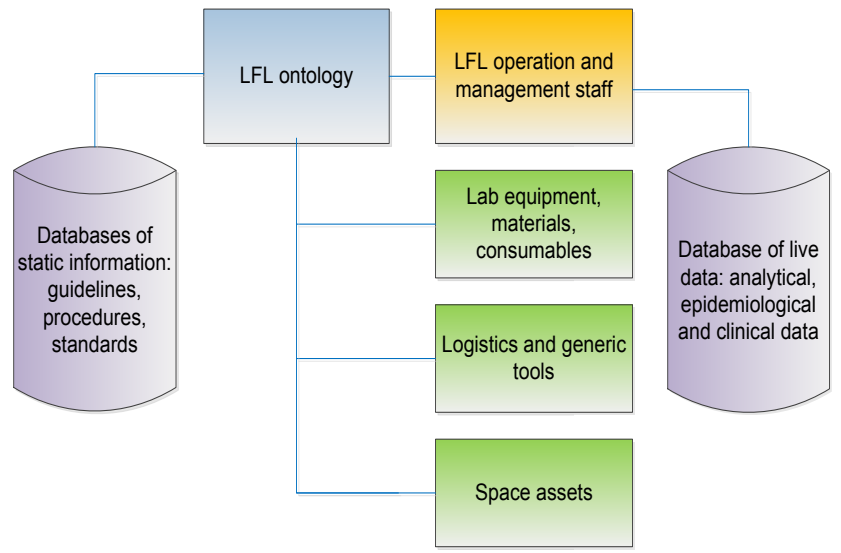

Figure 3. LFL Toolbox

The LFL provides a flexible and affordable working area for integrated equipment and systems that combine the advantages of current miniaturized and portable technologies. The challenge here is to take these tools and methods out of the reach back or reference laboratory facilities into a more demanding operational environment. To achieve this, tools, materials, and methods have to be selected, compacted and tested against field conditions where the main constraints will be the resistance to harsh operational conditions, biosafety, and easy on-site transportation. Once all the tools are transported and deployed on-site, the successful mission execution requires precise interoperability and good interconnection and communication between all components.
Technically speaking, the backbone of the B-LiFE system is the communication bus managing all data exchange performed by the B-LiFE system in order to deliver the service. The communication bus is the link for all communicating applications used in the LFL and for all communications inside and outside the LFL. The different components (IM system, Results Server, Lab Machines, Satellite maps and cartography, computers, phones, and other devices) are inter-connected. Whereas data from unmanned aerial system (UAS) was not part of the Ebola mission in Guinea, they are integral part of the B-LiFE system and will be used in future exercises, plainly justifying their inclusion in the current scheme.

Two ports are available for communication between LFL and "external world" - one is dedicated to communication with the sampling team and the other one to communicate with the headquarter laboratory and stakeholders (decision making agencies, authorities, international experts, etc.).

The data/information flow, the main interfaces and the protocols are depicted in Figure 4.

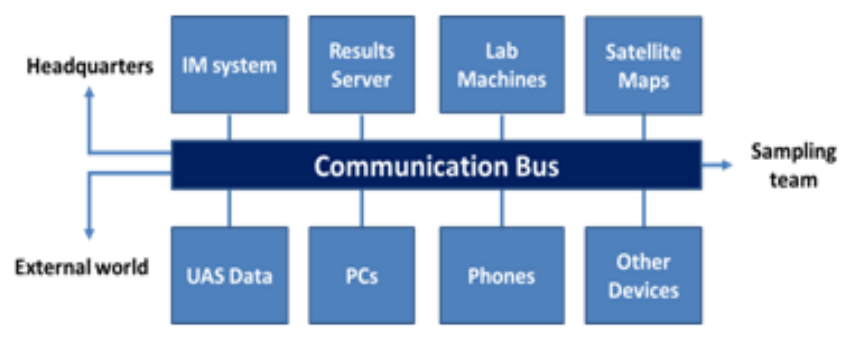

Figure 4. Information flow between B-LiFE components

The satellite communications-based capacity is designed for providing full system autonomy when deployed in a remote site where no local communication infrastructure is operational or is insufficient. Stable and reliable on-site communication (Wi-Fi and cell phone capacities connected to satellite terminals) provide all kinds of audio and/or visual communication through phones, e-mails, video conferencing that is vitally important for a successful and safe laboratory field operation. Indeed the information flow between all components of the deployed capacity, the sampling team in the field, the home-base laboratory, the direct beneficiaries and external stakeholders must be provided in real-time and maintained available at any time while minimizing as much as possible risks of interruption or breakdown. Results in the form of text files, graphical data, maps, tables, images and videos should be exchangeable through the whole chain of stakeholders. Analytical data from the LFL are transmitted by satellite telecommunication to secure servers. Data storage is implemented with the necessary ethical and legal consideration for sensitive data securing and for medical and personal data privacy.

Earth observation data and mapping are used both for the LFL deployment site selection before the mission and site monitoring on request afterwards. The Earth observation and geographical information system (EO/GIS) information flow is based on accurate and updatable cartographic data of the operational area with identification of relevant 
objects that can impact the capacity deployment. Dynamic demographic data, weather forecast and monitoring of the area for threats are transmitted to the LFL staff. High resolution UAS imagery can be used during missions to create new cartographic data for operational use. Integration of analytical data from the LFL with cartographic data provides capacities to create epidemiological maps denoting the affected areas and spread of contamination.

Geolocation data using navigation satellite systems is integrated in the information flow. Accurate geolocation data is used for refining the sampling strategy (i.e., determining where to take environmental samples of soil, dust, water, air), for guiding of the sampling team, and for tracking samples and equipment. The geolocation information is both used locally and is transmitted remotely.

Due to the integration of space assets, the information flow between the LFL operation and management staff, the sampling team and the local, regional, national and international stakeholders is reliably supported in real time in the framework of the emergency response capacity provided by B-LiFE. This advantage proved indispensable during the Ebola mission execution.

To unify the communication process at the document management level, the laboratory IM system (LIMS) is under development. Its use is deemed highly desirable as it facilitates many activities of the laboratory such as samples and analytical data recording, inventory control, quality management, data security and workflow efficiency [8 - 11]. An advantage of LIMS is that it can be integrated with the information systems of other organizations, e.g., other LFL and field hospitals, in order to harmonize the information sharing process and facilitate transmission of relevant data.

\subsection{Basis for Data Organization in the Ontology}

(a) OF implementation: In the LFL toolbox, different tools, i.e., technologies and processes, are associated to different operational functions (OFs). OFs are defined as activities (tasks) that need to be performed to identify and actively counter threats, to be prepared for, to respond to and to recover from incidents/attacks [7]. There are several conditions determining OFs implementation:

- When executing an OF, a particular goal/effect should be achieved;

- Particular resources will be needed to successfully perform a specific OF;

- Each OF is linked to other OFs serving as pre-conditions or triggers, being part of, being used in and linked to, as well as influencing, informing or referring to the given function;

- Thus advanced input/information is needed to start and successfully perform each particular OF.

The LFL components do not act separately but are unavoidably linked together by means of corresponding OFs to be executed within a single system (the LFL toolbox) in order to achieve the desired result of LFL work - to provide evidence-based decision support for decision-making authorities.

(b) OF organization: All OFs are grouped to perform a LFL mission cycle as depicted in Figure 5 below. Every OF is linked to other OFs by its properties, and is associated to the list of tools used to execute this OF. Such a system creates a single information space where all the components, OFs and tools must be described in a consistent way excluding redundancies and overlapping in order to be easily manageable. The LFL toolbox can be represented as a model consisting of a hierarchy of the classes, subclasses and individual concepts, and even more importantly the links between all the elements providing their usability within the toolbox.

(c) Operational requirements for LFL toolbox: In order to develop the LFL as an operational toolbox integrating technologies and processes, the following sub-objectives were pursued:

- To establish a mechanism allowing continuous improvement of the LFL structure and configuration;

- To identify and specify a mission statement for the LFL;

- To identify and describe the OFs required for carrying out the mission;

- To identify and describe the technologies and processes (tools) required for executing OFs;

- To develop the ontology to be applied to LFL missions preparation and to description of the relationship between OFs and tools;

- To test the performance of the LFL toolbox and the added value of the ontology as IM and decision support tool in on-site exercises.

(d) LFL toolbox and IM: The IM cycle in LFL corresponds to the principles previously described [1]: all the information collected about OFs, tasks, procedures, guidelines, best practices, tools and actors form the body of data that is then structured, processed and analyzed using the ontological approach [16]. This, in turn, facilitates the use of data by LFL operators, and by decision-makers, and provides complete, consistent, reliable, continuously updated and easily accessible data.

(e) LFL configuration: there are four types of missions implying a crisis situation and requiring a rapid LFL deployment:

(1) CBRN scenarios: The LFL mission consists of field assessment of the spread of environmental and human contamination resulting from a deliberate or accidental CBRN environmental contamination. The LFL mission is to provide evidence for accurate mapping of the indoor and/or outdoor contaminated area and persons at risk. This type of scenario is mainly considered within Europe. Main constraints are identified as rapidity of deployment, involvement of trained experienced staff, rapid analysis and identification of the threats, possibility of a mixed CBRN threat and biosafety issues for the staff and the population.

(2) Response to outbreak scenario: A typical example is an outbreak caused by a life-threatening and highly 
contagious agent in a remote area with insufficient local laboratory capacity; this type of crisis often affects low resources African countries. As illustrated for Ebola, a LFL is requested to provide quick and reliable diagnostic results for a longer period of time. The main constraints are the same as for CBRN scenario but remoteness, mission duration and harsh operational conditions imply extra-limitation of weight and volume, small size, robustness and easy handling of equipment, longer duration of the mission, and sustained maintenance and logistic support.

(3) Validation and use of new technologies on-site: This implies the selection and on-site testing of the most appropriate point-of-care testing (POCT) devices vs. conventional laboratory methods. The LFL mission is to validate new technologies in field conditions using the same type of samples. The main constraint is identified as the quality management of analytical procedures and results, a clear definition of the most appropriate biosafety procedures according to the type of B-agent, and the need for PCOT devices to withstand thorough, stringent and regular decontamination procedures.

(4) Training of local staffs and/or quality control of local capacities. The typical LFL mission is to contribute to the capacity rebuilding in a post-disaster situation where local laboratory infrastructure and local experimented staff are lacking. The main constraint is to adapt the work to the specificities of multicultural audiences, to take into account the lack of appropriate knowledge and training, and to rapidly evaluate local technological and analytical gaps.

Each category of LFL mission presumes execution of a range of OFs and a particular set of tools associated to the functions. Most OFs are activated for all types of missions, while the set of tools corresponding to very specific OFs are only applicable to particular missions. For example in the function PowerSupply, the tool Generator6,5kVa may not be required if the mission customer provides electricity, which is often the case for crisis in Europe.

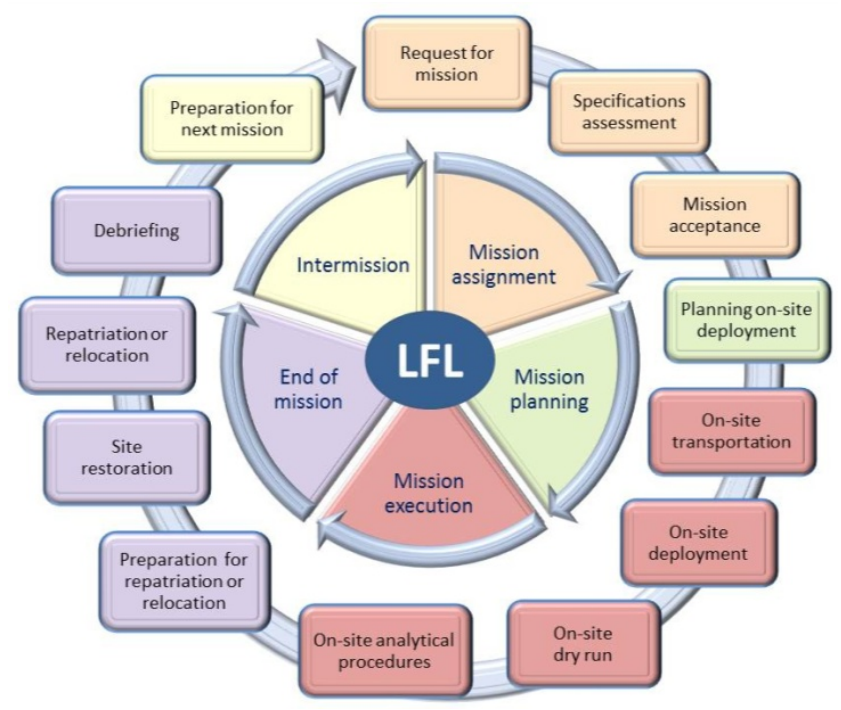

Figure 5. LFL Mission Cycle
The generalized LFL mission is represented as a cycle with 5 phases in 13 steps as described hereafter and illustrated by the Figure 5 .

Phase 1: Mission assignment starts with a request for mission addressed to the LFL service manager. The LFL manager needs to quickly evaluate this request, to check for mission feasibility and to assess mission parameters and specifications. The ontology-based IM system plays a major role in accelerating the whole process. The mission is confirmed as soon mission specifications are thought to in line with LFL capacity and available resources.

Phase 2: Mission planning starts as soon as the mission is confirmed. The LFL manager and staff prepare the mission for deployment according to the specifications of the mission. At the planning phase, the characteristics of on-site location where LFL will be deployed are defined and the LFL capacity is built according to the requirements of the mission; the plan for on-site access and installation are established, all authorizations and clearance required for staff and materials are collected, it is ensured that the host nation authorities are correctly informed about the mission, and provide their agreement to support it. The evaluation focuses on which services and tools can be supplied locally and which of them must be brought along. The costs of the mission are estimated and a tentative budget is established; the list of volunteers to participate in the mission is confirmed, the staff is comprehensively informed about the mission specifications. In addition to routine training during the intermission phase, complementary training is organized or specific extra-staff support is requested according uncovered needs (e.g., operating communication equipment, ensuring field safety and security recommendations, providing physical and technological training). All medical formalities, including health, eye and dental examination, vaccination and other preventive measures are fulfilled; on-site medical support and medical evacuation is planned with the stakeholders. In case clinical samples need to be collected during the mission, the required forms and approvals are proactively prepared in order to comply with and solve all ethical issues and guarantee personal data protection. The best suited LFL configuration is estimated and the list of tools to be transported is established; if necessary, useful complementary tools are purchased. All the tools are then packed and prepared for deployment. The duration of the planning phase mainly depends on the administrative and logistic constraints associated with acquisition of clearances and arranging transportation of staff, tools and logistics to the site of deployment according to international safety transportation rules (e.g., from the International Air Transport Association).

Phase 3: Mission execution is the core of the LFL mission cycle and therefore deserves a more detailed description. The mission execution starts when LFL staff and tools are transported to the deployment site. Accommodation is provided to LFL staff, the laboratory tent and tools are installed according to required operational conditions; healthcare and medical evacuation procedures 
are tested in case of necessity; on-site security is ensured. After having deployed the laboratory capacity, a dry run is carried out to test whether the capacity is fully operational. This includes ensuring that no power failure is observed when all equipment of the lab is running simultaneously and at full capacity; a check list enables LFL operators to verify the performances of each critical tool and procedure individually. Before starting to collect environmental or clinical samples, the pre-analytical phase is scrutinized to define the best and safest sampling strategy according to risk assessment, sample tracking (i.e., which sample collected where and, for biological samples, from whom), sample delivery by LFL sampling team or by third party, and sample reception by the LFL staff.

Reception of a sample requires a thorough recording of tracking data and assessment of sample packaging according to biosafety guidelines. Samples are then prepared and analyzed according to biosafety procedures; results are validated and interpreted.

Conventional support (printed or written results) and/or SatCom tools are used for real time transmission to pre-defined beneficiaries. Irrespective of the support, results are delivered in an unambiguous format that is fully informative while respecting European standards for data confidentiality, security and privacy. Follow up is ensured including availability of the staff for further information upon request and preparation of the LFL for the next analytical round. Waste is discarded and destroyed according to pre-defined safety guidelines and on-site capacity.

In Phase 4: The end of mission comprises the set of OFs dedicated to site restoration and preparation to LFL repatriation (return to the basis) or relocation (redeployment elsewhere). All equipment and materials used during the mission are cleaned and decontaminated before packaging; all temperature sensitive products are packed in appropriate coolboxes/containers for maintaining the cold chain unbroken; tools are prepared for transportation according to procedure (e.g., protection against shocks, removal or fixation of mobile parts, fuel is removed from power supply, etc.); tools are secured and safely loaded for transportation; repatriation takes place. Once back home or redeployed in another location, there is a debriefing step with immediate feedback on the successive phases of the mission just past. Return on experience is collected, lessons learned are drawn and a SWOT (strengths, weaknesses, opportunities and threats) analysis is made. If required a final report is prepared according for internal use and for specified stakeholders. A global listing of all analyses carried out during the mission is established and the in-depth inventory of all tools, samples and reagents used during the mission takes place. Tools which are not in good working order need to be repaired or replaced; waste not previously disposed on-site during past mission is discarded immediately according to biosafety rules. The total costs of the past mission is documented and updated. All tools are properly listed, repacked and stored for the next mission.
Phase 5: The mission cycle ends with the "Intermission" phase in which LFL capacity is continuously improved and prepared for a next mission. Survey of existing and emerging technologies, identification of future needs, identification of list of actions for improvement, acquisitions of new technologies and materials, development and implementation of new processes are all ongoing tasks. Lab processes, functions and materials are being continuously improved; staff undergoes regular training for optimally using new technologies, equipment and applying new processes. Competency of the staff is maintained and improved by regular exercises. All stocks of reagents are refilled and stored according to the shelf life. New sources of funding are identified for launching research and development projects according to the gap analysis during the debriefing step (see above End of mission phase) and for preparing for next missions.

\subsection{Development of an Ontology-based Description of the Relationships between Operational Functions and Tools}

LFL toolbox employs a knowledge base that is represented in a comprehensive ontology combining the tools, modelling patterns and a priori background knowledge about mission's parameters. The ontological approach to LFL description and mission's preparation serves a multifold purpose:

- To formalize and structure the domain of biological mobile laboratory operation in a crisis preparedness and response, therefore ensuring a continuous improvement of the performance;

- To provide an easy access to all the information, and to make the information reusable;

- To align the terminology, definitions between the tools and to provide a shared vocabulary of concepts to comply with the commonly recognized standards, best practices and procedures to facilitate common ground establishment between internal LFL operators and external decision- makers and other stakeholders;

- To provide technical and conceptual compatibility of sharable information between the heterogeneous tools.

The ontology here is a formal, explicit specification of a shared conceptualization describing the LFL as operational domain [12]. In computer science, information science, artificial intelligence and software engineering, an ontology defines a common vocabulary and provides a shared understanding and unambiguous interpretation of terms in the domain of interest, i.e., concepts, properties and attributes of concepts, constraints on properties and attributes, or individuals [13]. In computer science, ontologies are usually formalized by means of an ontology representation language that is both human- and computer-readable. This enables the ontology not only to be shared between different people (e.g., experts in the domain of interest and ontology developers), but also between different computer systems (e.g., a database and a decision 
support tool) or between humans and computers. Accordingly, the goal with an ontology is to make all specific knowledge of a domain computationally useful. Being formal and computer-readable, the ontology can then be validated [14]. In the current work, an ontology is therefore used as shared "standard" vocabulary for a domain with the purpose to integrate heterogeneous data sources, to annotate data with standardized terms for faceted browsing and for decision support. Regarding the LFL domain, the ontology expresses semantics of constructs in a formal language (e.g., OWL which is used in this presented work), enabling operators and developers to reason over the LFL domain constraints and properties, and to infer new facts from existing definitions.

In the this work the ontology describes concepts, their properties, relationships and constraints on relationships between concepts and properties, providing consistent and unambiguous modelling of LFL concepts, procedures, and functions which, in turn, helps differentiate functions which are necessary for the mission from those which are incompatible according to a given mission or scenario. This allows to follow the desired sequence and set of actions during the mission, and according to the desired procedure associated with a given mission profile, it allows to efficiently select only tools which are necessary and to activate them appropriately and in due time.

The LFL ontology is developed with the help of the open-source Protégé environment release 5.0.0 beta-17 [15]. In the current version the ontology comprises:

- 729 classes (including subclasses) describing the LFM mission cycles, steps, operational functions, sub-functions, 10 categories of 117 tools used for each function implementation;

- 58 individuals as members of classes denoting specific tools and materials used in the operational functions;

- 46 object properties that determine the relationships between functions, such as: one function triggering (an)other function(s), providing information and expertise, influenced by (an)other function(s), included, being part of and used in (an)other function(s), having particular parameters, e.g., type, time, source or location, etc.;

- 7 types of data properties that determine particular values of class members. These are specific values attributing to certain classes, such as values of temperature to be kept by the freezer, or predefined length of extension cords used in LFL.

All the information in the ontology can be both asserted (i.e., explicitly stated) and entailed by means of automatic reasoning. The logical consistency of the entire model of the LFL operation domain is ensured by 1348 logical axioms (rules) and filters delimiting the restrictions for all the relationships between all kinds of ontology entries.

The LFL tools used for executing various OFs consist of 10 groups with 117 categories:

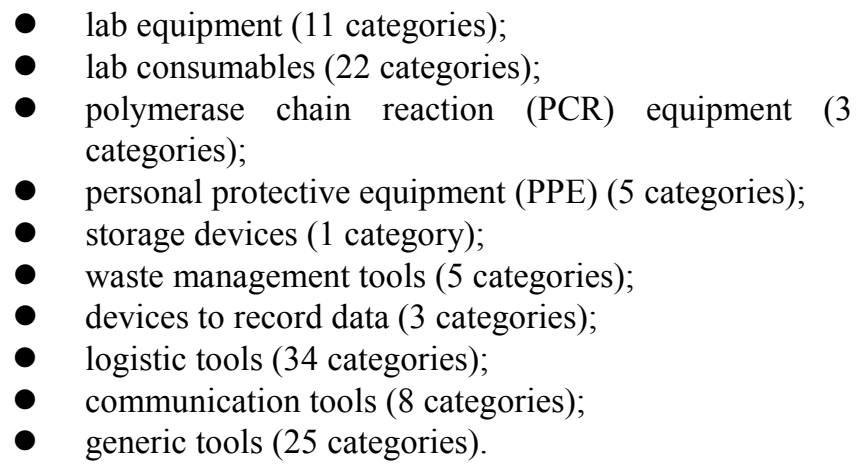

The major ontology classes describe the types of LFL missions, the LFL OFs that are performed during the different mission phases (confirmation, preparation, execution, mission site restoration, intermission), and the transversal OFs which are present in all cycles of LFL missions, as well as the tools used in OFs.

LFL operators described in detail the contents of OFs, the specific tasks within each function and the sequence of OFs within each mission cycle. Based on this knowledge, the relationships between all OFs were defined in the ontology. It is of note that OFs cannot be performed separately, but are all interrelated. For instance, the function SamplesReceptionAndValidationOfPackaging (in the MissionExecution cycle), at the high level cannot be executed unless LFL is deployed on-site with all corresponding OFs from LFL preparation to operation. At the direct level, it cannot be executed unless the Sampling has been made, thus SamplesReception function is triggered by several preceding functions. In turn, SamplesReception is linked to the function TransportationAnd DecontaminationOfSamples, which triggers the execution of the function ValidationOfPackaging. By nature, SamplesReception is linked to UpdatingRecordedData function, since tracking data linked with of every sample must be recorded to ensure a correct sample processing. The SamplesReception function can be associated with several tools by means of property employsTools and executed when these tools are used.

In the ontology, we manually denote only direct links between OFs which impact the close "neighbors". Each function invokes, in turn, other OFs and contains new parameters. The links between far distanced OFs are generated automatically by the system reasoner following the intrinsic logic of properties inheritance and extrapolation within and between classes of functions.

The whole map of all the multiple links between all operational functions is created automatically and can be visualized together or fragment by fragment for every function neighborhood. One such generalized fragment is illustrated below in Figure 6, showing a few links between OFs of MissionAssignment phase and MissionPlanning phase and other phases. Only major links are shown on this visualization, while secondary links are hidden for the sake of readability of this Figure. 


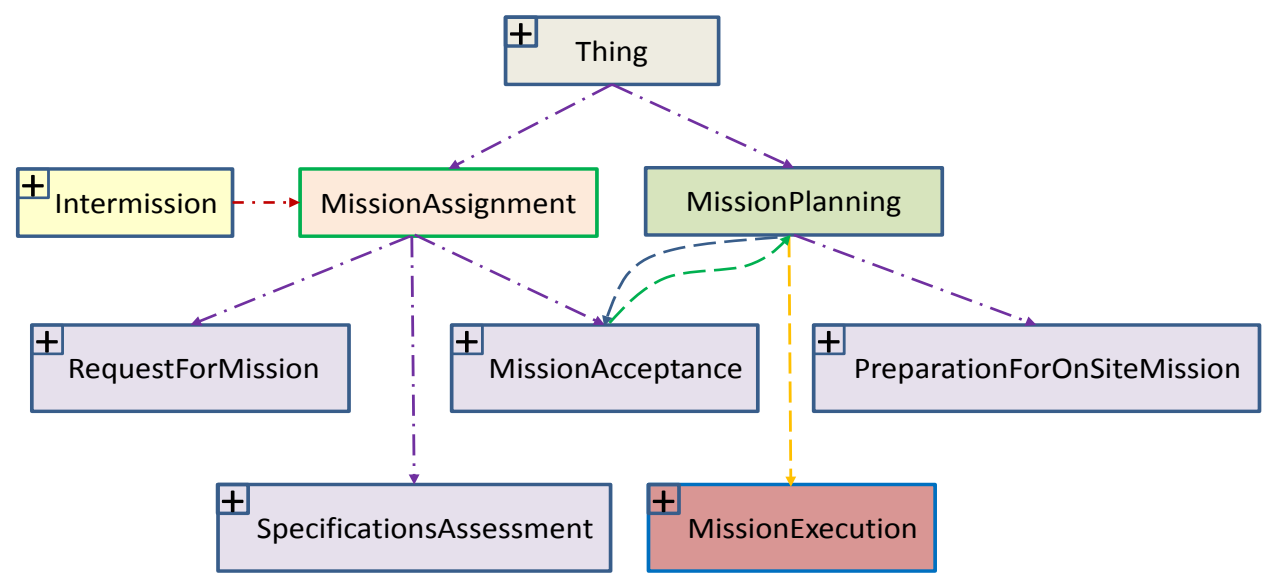

\begin{tabular}{l}
-1 - \\
“+”- - the node has other linked nodes \\
\hline
\end{tabular}

Figure 6. Fragment of ontology showing a few links between operational functions in the cycle MissionAssignment and MissionPlanning

\subsection{LFL Information Needs and Modeling of Decision-making Process}

The LFL staff fulfills three very distinct but interrelated roles. They are:

- Developers of bio-detection and bio-identification technology, where technology is usually developed and validated in the national fixed-based laboratory, and exported during on-site LFL operations;

- Operators (users) of LFL tools and equipment after development or taken off-the-shelf;

- Decision-makers, where the LFL manager has to take quick but highly critical and difficult decisions at each step of the LFL mission cycle.

Such interrelated roles are often shared at the same time by the LFL staff and manager and presume their ability to handle a wide spectrum of heterogeneous information which is collected from different sources and processed according to mission specifications. Likewise, the LFL IM system integrates mission specifications and reflects the interdependent LFL organizational structure. That is why the development and use of IM system as enabler of actualized information processing, communication and decision-making originates from on-site needs identified and formulated by LFL operators and decision-makers. Having analyzed the information needed by LFL staff, the following four main categories of information were considered relevant for a successful LFL operation:

(1) Geographical and political: This category contains general information which is a key parameter during decision-making and is part of first mission specifications;

(2) Organizational: For each of the 4 missions types described above (i.e., CBRN scenario, response to outbreak scenario, validation and use of new technologies on-site and training of local staffs and/or quality control of local capacities), this category includes practical mission specifications (also called mission parameters), types of actors (such as operators, beneficiaries of the results, policy-makers, etc.);

(3) Operational: This category depends on the information collected above (categories 1 and 2) the operational layer comprises all requirements, procedures, OFs and tools necessary for OFs execution.

(4) Dynamic situational awareness: It gathers information from all kinds of sources and feeds on-site decision support, among which are the location and size of affected area, local epidemiology of infectious diseases in the destination country and epidemiology of the current outbreak (i.e., the type of B-agent and its contagiousness, number of cases, gender, age, socio-economic status, occupation, medical condition at admission, mortality rate, outcome, and effectiveness of contact tracing). Treatment availability, efficacy, results from analyses leading to supportive, preventive and/or curative treatment are included here as well.

A successful mission implies collection of information in all four categories. Information of categories 1, 2 and 3 is collected from national and international partners and inner LFL sources. It is used to feed the LFL mission cycle and modeled in the ontology of which the rationale and structure are detailed above in Sections 2.2 and 2.3. The dynamic data of category 4 is contained in real-time updated databases used on-site where data are collected from national and local authorities, health providers and care takers, as well as from satellite communication and earth observation tools deployed within the B-LiFE system.

In the ontological data representation certain nodes act as 
decision centers and define what kinds of decision shall be made, while others act only as information centers the way of using that information for decision-making is defined by rules. In the present ontological hierarchy, the upper classes DecisionDescription, DecisionParameters, MissionAnd Service (including MissionType and MissionSpecifications), SubTypeOfService, SamplingStrategyParameters, and Actors model information of categories 1 and 2 and act as decision centers for high level decisions such as DecisionToDeployLFL, BiosafetyProcedures, Determine Costs, DecisionToEndMission. Among decision centers for higher level decisions, subclasses are decision centers for specific and nested decisions. For example, OFs such as DetermineCostsOfTransportation, DetermineCostsOf Equipment, DetermineCostsOfStaffOperation, are part of DetermineCosts. Likewise, the OFs Environmental Sampling, ClinicalSampling, AnimalsSampling specify DecisionToSample.

The information centers include all classes modeling the LFL mission cycle (i.e., MissionAssignment, MissionSpecification, MissionExecution, EndOfMission, Intermission, TransversalFunctions), as well as Tools containing description of OFs and associated tools. Classes like UserRequirements, SystemRequirements, Regulations AndStandards, LabInformationFlow contain reference data of category 3. The properties of data specified by these information centers, their relationships, the sequence and rules of their use in the decision-making process are modeled in the ontology.

It is important to emphasize here that in the presented ontological approach there is deliberately no distinction made between "right" or "wrong" decisions. In every on-site operation in a crisis situation, a series of urgent decisions need to be made based on a continuously actualized situational awareness. In this respect, the probability of dynamic and unexpected changes in a priori unstable operational environment should always be kept in mind. An operational deployment will therefore always seek to adapt to daily needs, constraints and issues. Consequently, daily decisions are successively taken throughout the LFL operation and will have short-term, mid-term and long-term impacts in terms of life-saving assistance to the population, problem solving and efficacy of decision-making by crisis managers.

In the absence of standards regulating the work of analytical capacities deployed on-site, there is a need for developing a harmonized approach which improves and facilitates the operational management irrespective of the type of LFL capacity [5, 18]. Based on lessons learned from the B-LiFE LFL involvement against Ebola in Guinea, the whole LFL mission cycle was reviewed and reshaped, leading to enhanced modeling of the decision-making process to improve preparedness for future missions.

At the higher level of decisions, a mission is extensively detailed in all its operational aspects according to mission specifications, including the type of B-agent which is essential in this process (see above category 4). The LFL configuration and set of tools needed for a particular mission both depend on the MissionSpecifications (acting as decision centers) modelled in the ontology as detailed hereafter:

MissionLocation: It can be Inside/OutsideEU. If the mission is OutsideEU, two further options are CountryWith DevelopedInfrastructure, or LowIncomeCountry. This parameter is linked to MissionDuration and LevelOfAutonomy which are parameters prescribing requirements for the amount and categories of equipment to be taken for the mission (e.g., autonomous power supply and autonomous communication means).

MissionDuration: It can be Short, MidTerm or Long. This parameter is crucial for preparing the most appropriate LFL configuration in terms of logistics, material, equipment and human resources. Accordingly, this parameter is linked to such parameters as MaterialsAndEquipmentNeeds, Logistics, Shelter, Communication, and NumberOfStaff NeededForMission.

NatureOfBiologicalAgent: As detailed previously in category 4 , this parameter is one of the most important one as it defines the specific set of tests required for diagnosing a specific disease, defining the level of Biosafety constraints, and possibly the need for SpecificStaffTraining.

NumberOfTests: This parameter is triggered by the MissionType to be performed; the LFL capacity depends directly on a total number of tests per day and during the whole mission; this is often based on a rough prior evaluation (planning) and needs therefore a constant adjustment according to the dynamics of the crisis. The mission type and number of tests are strong determinant of NumberOfStaffNeededForMission.

NumberOfStaffNeededForMission: This parameter is clearly related to MissionType, and MissionDuration parameters. A minimum of 3 staff members is foreseen when planning a short mission. Longer mission duration or any mission requiring additional expertise or staff support and more OFs will require a larger staff and/or staff turnover.

MaterialsAndEquipmentNeeds: This parameter depends directly on the MissionType, MissionLocation, MissionDuration, and LevelOfAutonomy; it is directly related to the Logistics parameter and to categories of Tools to be considered when preparing the mission.

LevelOfAutonomy comprises the options FullIndependency / UseOfLocalFacilities. These parameters also depend on MissionLocation, MissionDuration and MissionType and determine the MaterialsAndEquipment which are not provided locally by the host nation, and need therefore to be prepared and transported on-site.

PreparationTime can be Urgent, Middle or Planned. This is a parameter of MissionType specifying the degree of emergency, for instance the requirement of urgent deployment in response to a CBRN incident, the need for mid-term response in case of an outbreak, or a thoroughly planned intervention when the aim is to investigate on-site the spread of an endemic disease or when the LFL mission 
is focused on training its own or external laboratory staff. This parameter is related to the OFs in PlanningOnSite Deployment.

Logistics: This parameter includes AccommodationAnd Food, Transportation and depends on MissionLocation, MissionDuration and LevelOfAutonomy; all these parameters are used for Specifications Assessment.

CommunicationNeeds: this parameter depends on the LevelOfAutonomy, and is linked to CommunicationTools which are required according to the MissionType as well as SpecificationsAssessment.

Users are mainly understood here as beneficiaries and/or partners of LFL activity and results. They comprise Public, Private, and PublicPrivate entities. With regard to LFL, Users are those responsible for RequestForMission, they are partners or beneficiaries funding the mission, and are also involved in the MissionPlanning. Users for a particular mission can therefore be instantiated as individual members of the predefined categories, together with name, organization to which they belong and contact details.

Figure 7 hereafter illustrates the fragment of ontology linking LFLMissionAndService parameters:

The process of LFL ontology development faced a number of challenges among which differing perceptions between LFL staff members on the most appropriate organization of information in the LFL domain was key. For the purpose of ontology development, inputs were collected from experts (i.e., users). The collection was based on formulation and clarification of competency questions discussed individually and in group. Since it appeared that all users have different mental maps and different understanding regarding the interrelationship between all LFL components, it was challenging to achieve a consensus and validate the results. At first, a few variants of the data representation were considered while concurrently pursuing the analysis of user inputs. After several iterations, the current version of the data representation, relation, categorization and classification in the ontology could be proposed. However the development and validation of the OFs applied to LFL missions is still ongoing in the B-LiFE project. New specifications, amendments and clarifications of the ontological approach will result in a better understanding and identification of the different processes at stake through assessment of the ontology during successive LFL training exercises and missions.

Technically, the logical consistency of the ontology is being continuously verified using the OWL reasoner HermiT 1.3.8.3. plugged in Protégé 5.0.0 beta-17. Every time when a new entity (a class, an individual, a property, a rule, etc.) is introduced in the ontology or when relations between entities change, the reasoner automatically checks the whole logical structure for possible bugs, making sure that no errors occur that might cause output failure. Correct inference of every statement in the ontology guarantees desired answers to the users' queries, such as "What tools must be used to implement a certain OF?" or "Given a CBRN mission profile outside the EU with a mission duration of 3 weeks, what OFs shall be performed?"

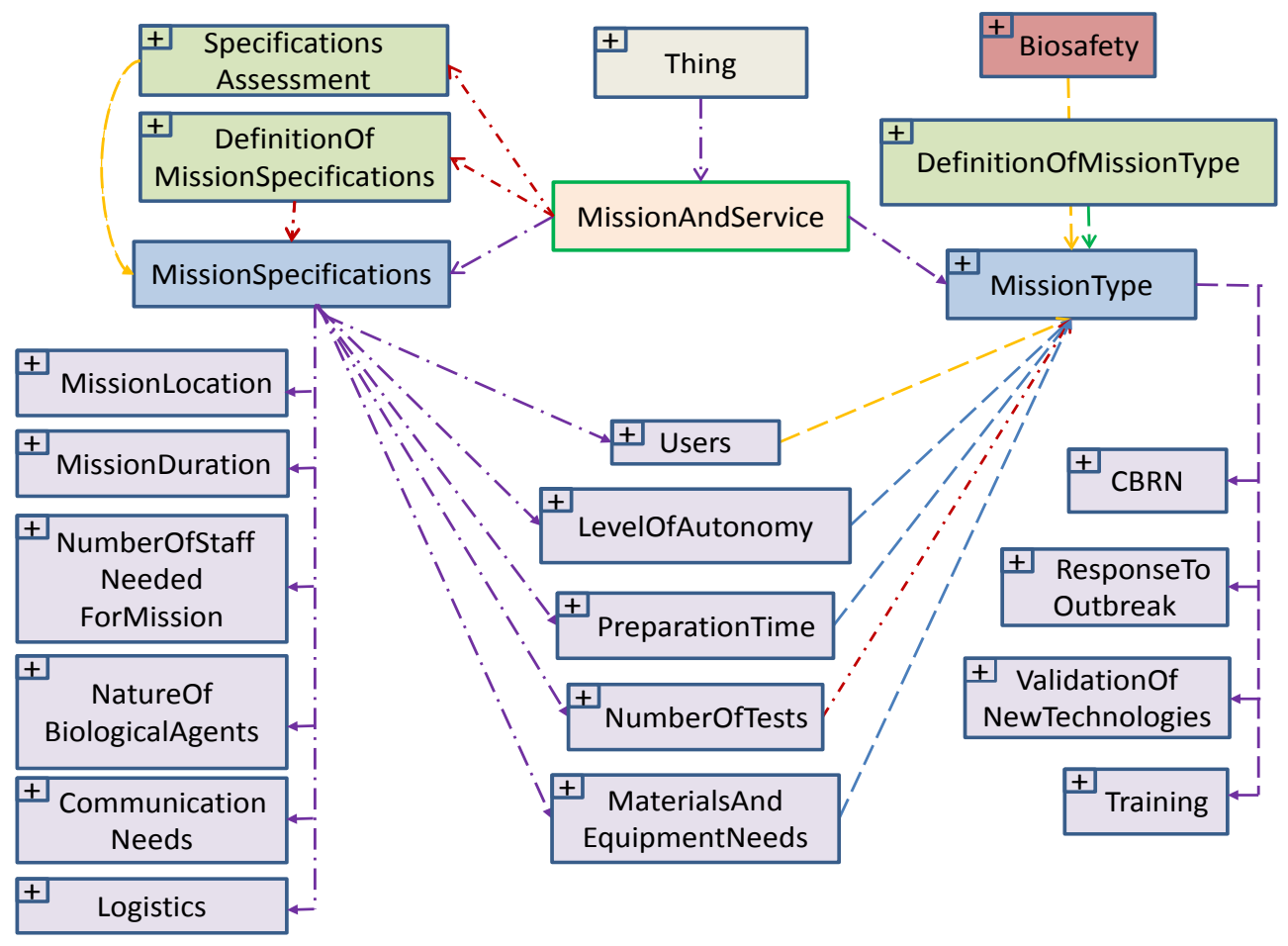

Figure 7. Fragment of the ontology linking LFL MissionAndService parameters 
The ontology, as currently developed, is being evaluated by LFL staff members according to the following performance parameters:

- Clarity: The transparency of knowledge representation is important to avoid misinterpretation, confusion, contradiction;

- Completeness: The ontology shall cover all the relevant concepts in the domain of interest without gaps in knowledge;

- Consistency: The ontology shall be free from any logical contradictions in the internal and external rules, definition of classes, subclasses, categories, concepts, terms, instances, their properties and interrelationships;

- Acceptability: The ontology shall consistently use the terminology commonly accepted and recognized both by developers and users; it will thoroughly and comprehensively describe the concepts, their attributes and interrelationships when consistent and relevant with the domain of interest.

For the moment, these parameters have been evaluated individually whereas the whole LFL ontology has been tested for users' acceptance and relevance by the entire LFL staff. The performance of LFL ontology has first been assessed through the use of CBRN scenario during the FP7-PRACTICE full scale international exercise PIONEX that took place in Pionki, Poland, in April 2014 [7]. The LFL concept was then tested in true operational conditions during the Ebola mission in Guinea and was updated and improved accordingly during the final debriefing and lessons learned phases.

A good usable ontology should not be a finite and frozen structure, but rather be a flexible tool which can be continuously updated and enriched by progressive introduction of additional classes, categories, concepts, terms, instances, properties and associated interrelationships according to new missions, broader expertise, and evolving state of the art. The future work will therefore include continuous assessment and performance improvement of the LFL ontology by exploiting new scenarios linked to any of the four categories of missions described above. In-house scenarios agreed by users will be developed. Solutions provided with and without the ontology will be compared.

The decision-making process will be further modeled in the ontology taking into account the ongoing risk assessment study. The risk factors and possible impacts of the risks associated with a specific mission as a whole and with execution of certain OFs in particular will be defined.

The formalized ontology-based mission planning and preparation is intended to provide a solid basis for development of a quality assurance manual. It also supports the systematic upgrade of LFL configuration and the performance of the whole IM system.

\section{Conclusions}

The biological LFL IM system aims at implementing an interdisciplinary approach to a whole set of LFL operational activities.

Integrating the LFL OFs with all their parameters and tools into a single information space managed by a single system strengthens the responders' operational and strategic capabilities.

The ontology has been developed with thorough consideration of the information needed by users and beneficiaries, so that it can rapidly and reliably provide answers to their questions and solved some of their issues. Harmonizing a concept of mobile laboratories that assemble different LFL capacities and a wide diversity of CBRN disciplines across many EU countries requires an ontological approach which was specifically addressed during the FP7-SEC MIRACLE project [5]. Structuring LFL data by means of mission cycle and OFs enables inventorying CBRN mobile laboratories in the EU and activating the best suited capacity according to mission specifications. Sharing and reusing this type of structured information is also needed when aiming to render existing LFL capacities more interoperable for joint training and operation, as ideally would be with the B-LiFE, the European Mobile Laboratory Project (EMLab) [17] and other national or international EU LFL capacities.

The use of a single ontology-based laboratory IM system facilitates the information flow and turnover of critical data between all components of the system. This undoubtedly has a substantial operational benefit in many crisis situations where there is a need to rapidly predict the unpredictable and where today's reality is different from what tomorrow will bring.

\section{Acknowledgements}

The B-LiFE project (Phase 1 - Feasibility Study, Phase 2 Demonstration Phase and Demonstration Phase CCN\#1 Ebola Mission) are funded by the European Space Agency in the framework of the IAP-ARTES 20 program. ESTEC contract $\mathrm{n}^{\circ} 4000105496 / 12 / \mathrm{NL} / \mathrm{US}$ and contract $\mathrm{n}$ 4000112330/14/NL/US.

The present work was supported by the project MIRACLE: Mobile Laboratory Capacity for the Rapid Assessment of CBRN Threats Located within and outside the EU, co-funded by the European Union's Seventh Framework Program for research, technological development and demonstration under Grant Agreement No. 312885 in 2013-2015, http://www.cbrnlab.eu/miracle/

\section{REFERENCES}

[1] The Operational Guidance Note on Information Management, 
2012

http://cpwg.net/wp-content/uploads/sites/2/2014/07/OCHAWhat-IM-Guidelines-ENG.pdf.

[2] M. Turoff, M. Chumer, B. Van de Walle, X. Yao: The design of a dynamic emergency response management information system. Journal of Information Technology Theory and Applications, 5(4), 1-36, 2004.

[3] B. Van de Walle, B., T. Comes: Risk Accelerators in Disasters. Insights from the Typhoon Haiyan Response on Humanitarian Information Management and Decision Support. In M. Jarke, J. Mylopoulos, \& C. Quix (Eds.), CAiSE2014 (pp. 12-23). Thessaloniki, Greece: Springer Berlin / Heidelberg, 2014.

[4] T. Comes, O. Vybornova, B. Van de Walle: Bringing Structure to the Disaster Data Typhoon: an Analysis of Decision-Makers' Information Needs in the Response to Haiyan // In: Proceedings of the AAAI Spring Symposium Series (SSS-15) on Structured Data for Humanitarian Technologies: Perfect Fit or Overkill? March 23-25, 2015, Palo Alto, CA, USA.

[5] O. Vybornova, JL Gala et al.: CBRN Mobile Laboratories. FP7-SECURITY MIRACLE project (2013-2015) Mobile Laboratory Capacity for the Rapid Assessment of CBRN threats Located within and outside the EU: Major Recommendations. http://www.cbrnlab.eu/miracle/.

[6] C. Dumont, L. Irenge, E.K. Magazani, D. Garin, JJ.T. Muyembe, M. Bentahir, JL Gala: Simple technique for in field samples collection in the cases of skin rash illness and subsequent PCR detection of orthopox viruses and varicella zoster virus. In: PloS one, Vol. 9, no.5, p. e96930, 2014 http://dx.plos.org/10.1371/journal.pone.0096930.

[7] FP7-SEC PRACTICE (2011 - 2014). Preparedness and Resilience against CBRN Terrorism using Integrated Concepts and Equipment. Grant agreement $n^{\circ}: 261728$ http://www.practice-fp7-security.eu/.

[8] H. Berger, Laboratory Information Management Systems Challenges of implementation, 9th International Conference Knowledge Management in Organizations, 281-287, 2014.
[9] D.O. Skobelev, T.M. Zaytseva, A.D. Kozlov, V.L. Perepelitsa, and A.S. Makarova: Laboratory Information Management System in the work of the analytic laboratory. Measurement Techniques, Vol. 53, No. 10, 1182 -1189, 2011.

[10] HL7 Healthcare Privacy and Security Classification System (HCS). Online available from

http://www.hl7.org/implement/standards/product_brief.cfm? product_id=345.

[11] WHO, Laboratory quality management system handbook, WHO Library Cataloguing-in-Publication Data, 2011.

[12] O. Vybornova, PA Fonteyne, JL Gala: Ontology-Based Knowledge Representation and Information Management in a Biological Light Fieldable Laboratory // Long paper: proceedings of the Conference Information Systems for Crisis Response and Management (ISCRAM 2015), May 24-27, Kristiansand, Norway.

[13] T. Tudorache. Ontologies in Engineering: Modeling, Consistency and Use Cases. VDM Verlag Dr. Mueller, Saarbruecken, Germany, 2008, ISBN 10: 3639049799, ISBN 13: 9783639049794.

[14] T. J. Grant: Formalized Ontology for Representing C2 Systems as Layered Networks. // In: Network Topology in Command and Control: Organization, Operation, and Evolution / [ed] T. J. Grant, R. H. P. Janssen, \& H. Monsuur, Hershey, PA: IGI Global, 2014.

[15] Protégé. A free, open-source ontology editor and framework for building intelligent systems. Online available from http://protege.stanford.edu/.

[16] Decision-makers taxonomy V1, August 2013. UN Office for the Coordination of Humanitarian Affairs, Digital Humanitarian Network. Online available from http://reliefweb.int/report/world/decision-makers-taxonomy.

[17] European Mobile Laboratory Project http://www.emlab.eu/.

[18] AS Piette, O. Vybornova, M. Bentahir, JL Gala: CBRN: detection and identification innovations. Crisis Response Journal; Vol 10, Issue 2, 36-38, 2014. 